\title{
THE ROLE OF RADIATION PRESSURE IN LBV ATMOSPHERES
}

\author{
I. APPENZELLER \\ Landessternwarte Königstuhl \\ D-6900 Heidelberg 1 \\ Federal Republic of Germany
}

\begin{abstract}
As LBVs have luminosities close to their Eddington limits, their structure is profoundly influenced by radiation pressure. Radiation pressure effects probably cause the highly extended atmospheres and the extreme mass loss observed during the maximum states of the S Dor variables. An opacity-related instability of the radiative acceleration combined with a delayed thermal readjustement of the subatmospheric layers possibly explains the large-amplitude radius variations of these objects.
\end{abstract}

\section{Introduction}

The number of mechanisms which have been suggested in order to explain the variability of LBVs probably exceeds the number of reliably identified members of this exciting class of stars. Nevertheless, in view of the observed very high luminosities, everybody seems to agree that radiation pressure effects have to be taken into account and play a major role in realistic models of these stars. As noted by Humphreys and Davidson (1979), Lamers (1986a), Davidson (1987), Lamers and Fitzpatrick (1988), and others, radiation pressure certainly contributes to the exceptionally strong mass loss of the LBVs. Furthermore, instabilities of the radiative acceleration in the expanded, nonstatic atmospheres of the LBVs have been suggested to cause the observed strong variability (Appenzeller 1986, Lamers 1986b). However, in the absence of detailed time dependent model atmosphere computations, other variability mechanisms (see e.g. Stothers and Chin 1983, de Jager 1980, 1984, Maeder 1983, and various contributions to this conference) cannot be ruled out. Hence, this review will be separated into two parts. I shall start with a brief summary of the relatively well established radiation pressure effects in equilibrium atmospheres. This will be followed by a more speculative discussion of the opacity-related radiation pressure instabilities and of a relaxation oscillation scenario of the the observed variability. 


\section{Equilibrium States and Eddington Limit}

The atmospheric equilibrium of a nonrotating, nonmagnetic, nonturbulent luminous star can be described by the equation of motion

$$
\frac{d v}{d t}+v \frac{d v}{d r}+\frac{1}{\rho} \frac{d P_{G}}{d r}=-g_{e f f}=-g\left(1-\frac{g_{R}}{g}\right)
$$

where $\mathrm{v}=d r / d t$ is the flow velocity, $\mathrm{t}$ the time, $\rho$ the density, $P_{G}$ the gas pressure , and $g$ and $g_{R}$ denote the gravitational and radiative accelerations, given by

$$
\begin{gathered}
g=\frac{G M}{R^{2}} \\
g_{R}=\frac{4 \pi}{c} \int \kappa_{\nu} H_{\nu} d \nu
\end{gathered}
$$

$\left(\mathrm{M}=\right.$ stellar mass, $\mathrm{R}=$ stellar radius, $\kappa_{\nu}=$ mass absorption coefficient, $H_{\nu}=$ Eddington flux).

Among the various types of solutions of Equ. (1) are the following well known special cases: For $v \equiv 0$ Equ. (1) degenerates to the hydrostatic equation which describes "normal" static stellar atmospheres. As shown by Equ. (1), for $g_{R} \geq g$ no such static solutions are possible. $d v / d t \equiv 0$ and $v d v / d r>0$ corresponds to the case of stationary accelerated stellar winds. Finally, we note that for $g_{\text {eff }}=$ $g\left(1-g_{R} / g\right) \rightarrow 0$ the pressure (and density) scale height of hydrostatic models

$$
h=\frac{\Re T}{\mu g_{\text {eff }}}
$$

$(\Re=$ gas constant, $\mu=$ mean molecular weight) approaches infinity. Hence, with increasing radiation presure effects atmospheres become progressively more extended.

In the idealized case of a gray atmosphere, i.e.

$$
\kappa_{\nu}=\text { const. }
$$

we have

$$
g_{R}=\frac{4 \pi \kappa_{\nu}}{c} \int H_{\nu} d \nu=\frac{\kappa_{\nu} L}{4 \pi c R^{2}}
$$

and thus

$$
\frac{g_{R}}{g}=\frac{\kappa_{\nu} L R^{2}}{4 \pi c R^{2} G M}=\frac{L}{L_{E D D}}
$$

where $\mathrm{L}$ is the stellar luminosity and

$$
L_{E D D}=4 \pi c \kappa_{\nu}^{-1} G M
$$

is called the "Eddington luminosity" or "Eddington limit". For very hot stars, where the opacity is dominated by Thomson scattering we, have for a Population I chemical composition

$$
L_{E D D}=3.910^{4} \frac{M}{M_{\odot}} L_{\odot}
$$


Using the luminosity values presented at this conference in the reviews of $R$. Humphreys and B. Wolf and using stellar mass values derived by matching these luminosities to the evolutionary tracks published by Maeder and Meynet (1987), we obtain for the known S Dor LBVs electron-scattering $L / L_{E D D}$ ratios between 0.5 (R71) and 0.8 (R127). In view of the fact, that pure electron scattering is expected to underestimate the opacities of real LBV atmospheres, these values are surprisingly high and confirm the importance of radiation pressure for these objects.

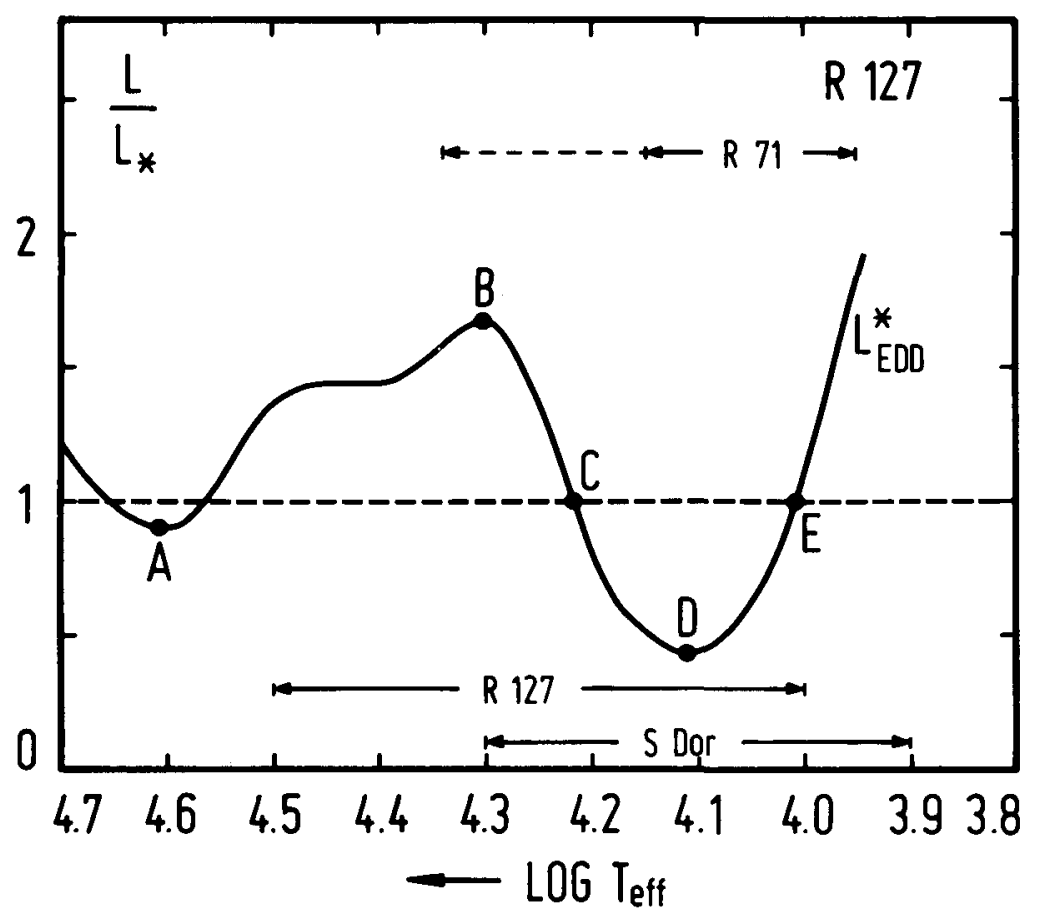

Figure 1: Approximate temperature dependence of the photospheric modified Eddington luminosity of a bright LBV. Also shown are the $T_{e f f}$ ranges of three LMC S Dor variables.

In a real LBV atmosphere $L_{E D D}$ has no direct physical meaning as the absorption coefficient depends on the frequency. However, locally the radiation pressure effects can still be characterized by the ratio $g_{R} / g$ or by the "modified Eddington luminosity" $L_{E D D}^{*}$, defined (analogous to Equ.(7)) by

$$
L_{E D D}^{*} / L=g / g_{R}
$$

In contrast to the conventional Eddington luminosity $L_{E D D}$, which is constant within an atmosphere, $L_{E D D}^{*}$ normally is a function of the optical and geometrical depths. But, if the atmospheric structure of a star is known, for each depth $L_{E D D}^{*}$ can be calculated using Equs. (3) and (10). 


\section{Instabilities}

In realistic atmospheres $\kappa_{\nu} H_{\nu}$ and thus $g_{R}$ and $L_{E D D}^{*}$ depend on the effective temperature. A very approximate plot of this dependence for the photosphere of a bright LBV, such as R127, is given in Figure 1. In the absence of more suitable model grids, the plotted relation $L_{E D D}^{*}\left(T_{\text {eff }}\right)$ has been based on the functions $g_{R}\left(T_{\text {eff }}\right)$ computed by Kurucz and Schild (1976) on the basis of hydrostatic LTE model atmospheres. As LTE atmospheres are expected to introduce in the case of LBVs a qualitatively incorrect dependence of the line opacity on the gravitational acceleration, the $\mathrm{g}$ dependence of $\kappa_{\nu}$ was neglected. While the static Kurucz and Schild models are probably reasonable approximations for the slowly expanding atmospheres of S Dor variables, they are unrealistic for high wind velocity $O$ stars. Hence, at high tempreatures Figure 1 becomes a progressively poorer approximation. Using the more recent line acceleration values (based on non-static atmospheres) tabulated by $\mathrm{Ab}$ bott (1982) gives qualitatively similar results. But, as many weak lines, which are important at the low temperature of the $\mathrm{S}$ Dor variables at minimum, were not included in the nonstatic computations, the Kurucz and Schild models are probably the more adequate approximation for the present purpose.

The most important feature of the $L_{E D D}^{*}\left(T_{\text {eff }}\right)$ relation is a conspicuous minimum near $\log T_{\text {eff }}=4.1$, followed by a steep increase for lower temperatures. This minimum of $L_{E D D}^{*}$ and the corresponding maximum of the radiative acceleration is mainly due to a large number of merging lines of singly ionized iron group elements (Fe II, Cr II,...) occuring near the maximum of the stellar flux distribution (Kurucz and Schild 1976). The great importance of these lines for LBV atmospheres is evident from Figure 2.

For a radiation pressure dominated stellar atmosphere Figure 1 predicts a dynamical instability for $4.1 \leq \log T_{\text {eff }} \leq 4.3$ (Points $B$ to $D$ ). In this range we have $d L_{E D D}^{*} / d T_{\text {eff }}>0$. Hence, any increase of the photospheric radius (or mass loss) at constant luminosity and the corresponding decrease of the effective temperature results in a increase of $g_{R} / g$, a lowering of $g_{e f f}$, and thus in a further expansion and further increased mass loss. Thus, any radius or $T_{\text {eff }}$ perturbation results in a runaway. Outside this instability range (except for the region to the left of $A$ where the static LTE models are not applicable) Figure 1 indicates dynamical stability, since perturbations will result in a return to the initial equilibrium state.

A sufficiently massive post-main sequence star, evolving with approximately constant luminosity to the right in the HR diagram, will sooner or later encounter the instability outlined above. At a critical effective temperature (which, because of the relation between opacity, gravity, and atmospheric chemical enrichment, will depend somewhat on the initial mass) the photosphere will lose hydrostatic equilibrium and the star will rapidly move to lower effective temperatures, until the two conditions $d L_{E D D}^{*} / d T_{\text {eff }}<0$ and $L_{E D D}^{*}>L$ are both fulfilled again. According to Figure 1, the region where LBVs are expected to regain equilibrium coincides well with the observed minimum-state temperature of the $\mathrm{S}$ Dor variables, while the maximum-state temperatures are (at least for $S$ Dor and $R$ 127) compatible with a location on the 
S Dor LWR 15331 1983, Feb. 19
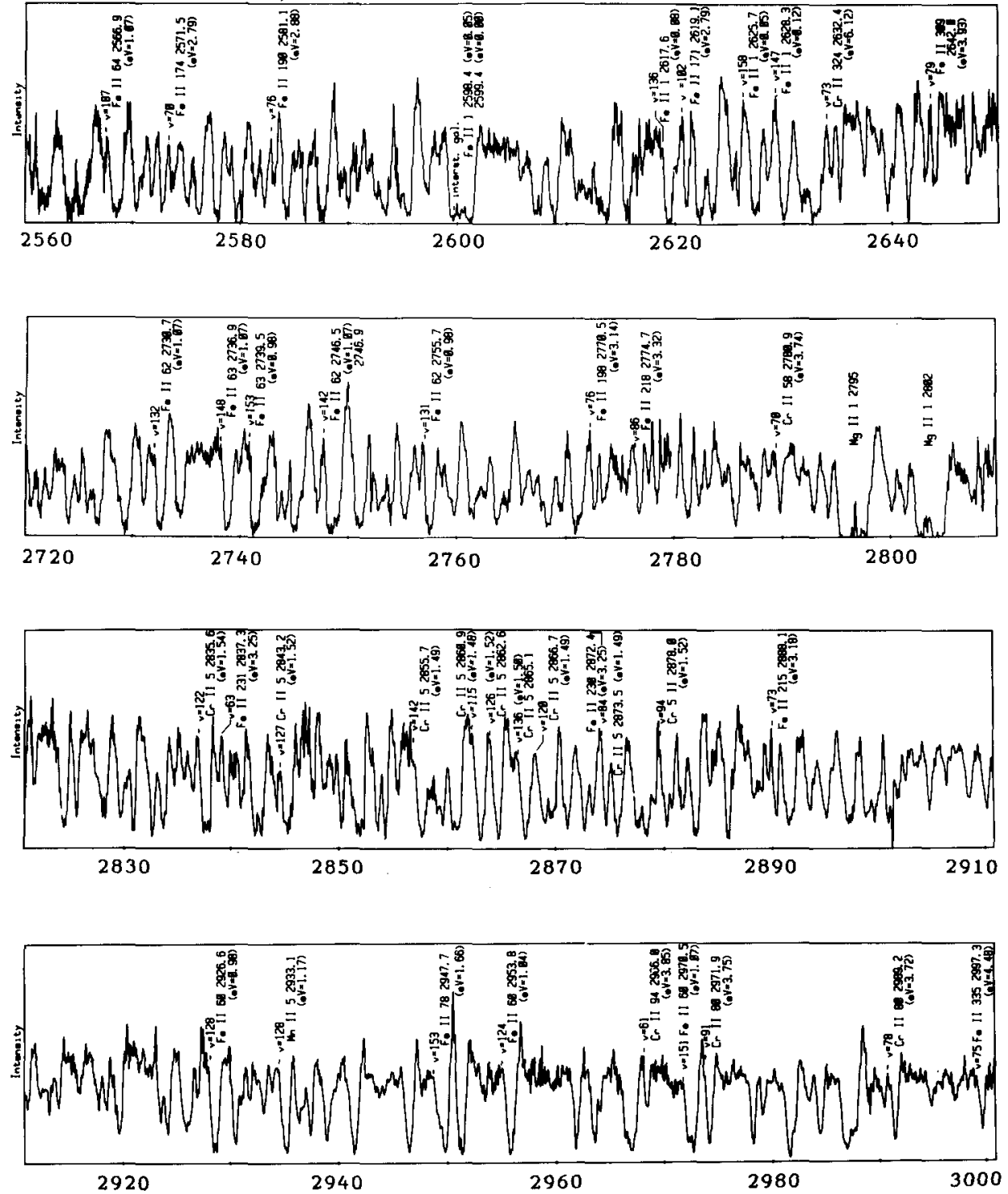

Figure 2: Sections of the $2500-3000 \dot{A}$ spectrum of $S$ Dor at maximum. Note the large number of merging absorption features of singly ionized iron group elements. (From Leitherer et al. 1985). 
hot stable branch of the $L_{E D D}^{*}\left(T_{\text {eff }}\right)$ relation. Hence, it seems attractive to identify the extended maximum and minimum states of the $S$ Dor variables with the two different stable configurations predicted (qualitatively) by Figure 1. However, while the above scenario allows to understand the transition from the minimum state to the maximum state and the observed properties of the two states, additional physical processes are needed to cause the observed return (after $\sim$ a few decades) from the stable maximum state to the high $T_{\text {eff }}$ minimum state. An example for such a process is discussed in the following section.

\section{The Relaxation Oscillation Scenario}

The maximum and minimum states of $S$ Dor stars are characterized by highly different effective temperatures and effective $(\tau=2 / 3)$ radii. On the other hand, the bolometric luminosity remains essentially constant (Appenzeller and Wolf 1981, Stahl et al. 1983, Humphreys et al. 1984, Wolf, 1986, 1987). This indicates that the $S$ Dor variability is mainly an effect of the outer stellar layers. However, as illustrated by Figure 3, for these outer layers the change is dramatic. In the case of $\mathrm{S}$ Dor the increase of the effective radius amounts to a factor 7 . For R127 the corresponding ratio is about 15 .

\section{S DOR}

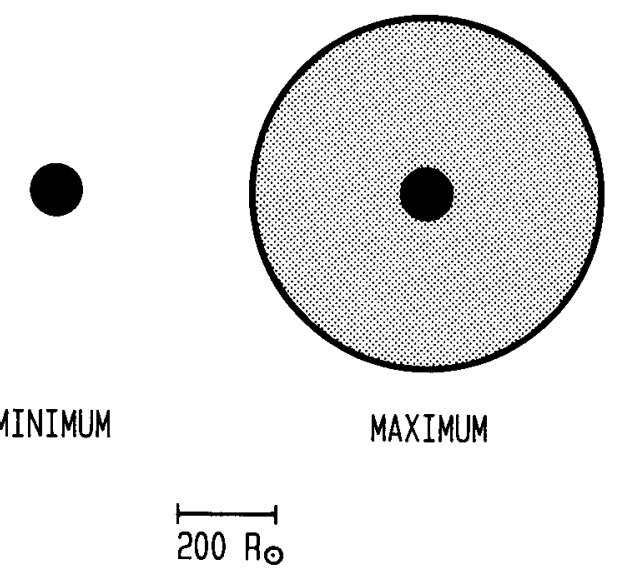

Figure 3: Comparison of the effective $(\tau=2 / 3)$ radii of $S$ Dor at minimum and maximum.

From the wind models one can estimate that the highly expanded nonstatic layers contain about $10^{-4}$ of the stellar mass. For the deeper interior the envelope expansion is practically equivalent of (temporarily) removing this mass on a dynamical timescale. Hence the interior will react with a fundamental mode radial expansion. 
Because of the central mass concentration of supergiants, the relative amplitude will be small in the deep interior. $\Delta r / r$ will be above 0.1 only for about the outermost $\sim 3$ percent of the mass.

The question of how a star reacts to the removal of mass from its surface has been investigated thoroughly in the context of mass-exchange binary evolution (Morton 1960, Kippenhahn and Weigert 1967, Plavec 1970, Webbink 1977, Heisler and Alcock 1986). As noted by these authors, a star's reaction depends on the ratio between the mass-loss time scale and the thermal adjustment time

$$
\tau_{K H}=\frac{G M^{2}}{R L} \approx 3.110^{7} \frac{\left(M / M_{\odot}\right)^{2}}{\left(R / R_{\odot}\right)\left(L / L_{\odot}\right)} \text { years }
$$

which for typical LBVs is of the order $\tau_{K H} \approx 10^{3}$ years. But, as pointed out by Kippenhahn and Weigert (1967) for stellar surface layers we may have much shorter local thermal adjustment times. A surface layer of mass $\Delta m$ is expected to regain thermal equilibrium after about

$$
\tau_{\Delta m}=\frac{G M \Delta m}{R L}
$$

For the outermost $10^{-4}$ of the stellar mass $\tau_{\Delta m}$ corresponds roughly to its dynamical time scale $(\approx \mathrm{a}$ few weeks). Hence, these layers are expected to remain in thermal equilibrium at all times. However, as noted above, the atmospheric expansion will have a significant effect for the subatmospheric layers down to about $M_{r} / M \approx 0.97$. For this whole volume Equ. (12) indicates a thermal adjustment time of several decades (i.e. of the order of the duration of the minimum and maximum states of $S$ Dor variables). Consequently, these subatmospheric layers expand adiabatically and lose thermal equilibrium. The resulting thermal adjustment requires additional heating of the expanded gas. Hence, a local minimum of the heat flow is expected to occur somewhere in these outer layers. Below the minimum the gas will slowly expand, above the minimum initially a slow contraction will occur. Because of the larger volume of the outer layers, the net effect will probably be an initial net contraction and heating of the subatmospheric layers. Obviously, such a contraction and heating on a thermal time scale could cause the eventual collapse of the extended atmosphere, resulting in a return to the hot minimum state. The collapse of the atmosphere is again expected to occur on the shorter dynamic timescale. Hence, the new minimum state will also be out of thermal equilibrium, resulting in a new thermal adjustment, now probably accompanied by a slow expansion, until the atmosphere again encounters the radiation pressure instability and a new cycle of the relaxation oscillations is started.

In principle, the scenario described above can explain all basic properties of the observed variability of the $\mathrm{S}$ Dor stars. However, so far the above suggestions are based on qualitative considerations, very rough numerical estimates, and on the analogy to computed models of mass loosing binary components. To confirm or disprove the above scenario, selfconsistent time-dependent non-static models of LBV outer layers (including the winds, atmospheres and the optically thick subphotospheric layers) 
are needed. Impressive progress in computing stationary nonstatic LBV models has been reported at this meeting by Kudritzki (1988) and by Leitherer and Abbott (1988). An extension of such computations to time dependent cases will allow a quantitative study of the radiation pressure related instabilities. But, already from the qualitative discussion outlined above it is clear that radiation pressure effects play an important but complex role in the LBV atmospheres.

\section{References}

Abbott, D.C.: 1982, Astrophys.J 259, 282

Appenzeller, I.: 1986, IAU Symp. 116, Luminous Stars and Associations in Galaxies C. W.H de Loore et al. eds.,p. 139

Appenzeller, I., Wolf B., 1981, in "The Most Massive Stars", S. D'Odorico et al. eds., ESO Proceedings, Garching 1986, p. 131

Davidson, K., 1987, Astrophys. J. 317, 760

de Jager, C., 1980, The Brightest Stars. D. Reidel, Dordrecht 1980

de Jager, C., 1984, Astron. Astrophys. 138, 246

Heisler, J., Alcock, C., 1986, Astrophys. J. 306, 166

Humphreys, R. M., Davidson, K., 1979, Astrophys. J. 232, 409

Humphreys, R. M., Blaha, C., D'Odorico, S., Gull, T.R., Benvenuti, P., 1984, Astrophys. J. 278, 124

Kippenhahn, R., Weigert, A., 1967, Zeitschr. Astrophysik 65, 251

Kudritzki, R. P., 1988, elsewhere in this volume

Kurucz, R. L., Schild, R. E., 1976, IAU Symp. 70, Be and Shell Stars, A. Slettebak ed., p. 377

Lamers, H. J. G. L. M., 1986a, Astron. Astrophys. 150, 90

Lamers, H. J. G. L. M., 1986b, IAU Symp. 116, Luminous Stars and Associations in Galaxies, C.W.H. de Loore et al. eds., p. 157

Lamers, H. J. G. L. M., Fitzpatrick, E.L., 1988, Astrophys. J.324, 279

Leitherer, C., Appenzeller, I., Klare, G., Lamers, H. J. G. L. M., Stahl, O., Waters, L. B. F. M., Wolf, B., 1985, Astron. Astrophys.153, 168

Maeder, A., 1983, Astron. Astrophys. 120, 113

Maeder, A., Meynet, G., 1987, Astron. Astrophys. 182, 243

Morton, D. C., 1960, Astrophys. J. 132, 146

Plavec, M., 1970, Publ. Astron. Soc. Pacific 82, 957

Stahl, O., Wolf, B., Klare, G., Cassatella, A., Krautter, J., Persi, P., Ferrari-Toniolo, M., 1983, Astron. Astrophys. 127, 49

Stothers, R., Chin, C., 1983, Astrophys. J. 264, 583

Webbink, R. F., 1977, Astrophys. J. 211, 486

Wolf, B., 1986, IAU Symp. 116, Luminous Stars and Associations in Galaxies, C.W.H. de Loore et al. eds., p. 151

Wolf, B., 1987, IAU Symp. 122, Circumstellar Matter, I. Appenzeller and C. Jordan eds., p. 409 


\section{DISCUSSION}

Owocki: If the radiative forces everywhere exceed gravity, then this does not imply a steady-outflow wind, because that would require a subsonic region that is fixed at the base. I think the situation implies instead that there must be a time-dependent, not steady, mass outflow. I believe then that we must begin to address this time dependence explicitly. With some reasonably simplified formulation for the opacity effects that you have described, this is quite feasible to do numerically.

Appenzeller: In the interior layers of LBV's we have $\left|g_{\mathrm{rad}}\right|<g_{\mathrm{grav}}$. But time-dependent model computations are clearly desirable.

Kudritzki: A comment to Stan Owocki's remark. I totally agree: time-dependent calculations for simplified or parametrized wind line opacities are important and can be done. We plan to do such calculations as well. This was why yesterday, on the river, I was so anxious that Stan should find his way back into the raft ...

Langer: LBV's are probably in global thermal disequilibrium: the H-burning shell continuously adjusts as the core contracts toward helium ignition. This may be a continuous cause of envelope expansion on a thermal time-scale $\sim 10^{3} \mathrm{y}$.

Appenzeller: Yes. But thermal readjustment of just the outer layers following outbursts will have a time-scale $\sim 10 \mathrm{y}$.

Maeder: Is it possible to estimate the total amount of mass ejected in one of the events that you described in the framework of the radiation pressure model?

Appenzeller: An order-of-magnitude estimate is $\dot{M} \times$ duration $\sim 10^{-2} M_{\odot}$. Much higher values (as for $\eta$ Car) could be achieved only by radiation pressure effects at high optical depths, $\tau \gg 1$.

Walborn: Does the bulk of the material producing the A-type maximum spectrum (false photosphere, shell) leave the star or does it fall back?

Appenzeller: About $10^{-2}$ of the stellar mass is affected by the expansion. $\dot{M}$ is not large enough to remove this much matter during the eruption. So most of the expanded layers are expected to contract again.

Wolf: $\mathrm{R} 127$ starts to get unstable at a temperature higher than expected in your diagram of the modified Eddington limit. In my talk I showed that Ofpe/WN9 stars have enhanced absorption lines, e.g. Fe III originating from metastable levels and even Al III lines, normally unexpected in $\mathrm{O}$ stars. Can this additional line opacity make these more luminous stars radiatively unstable at higher temperature?

Appenzeller: Although Fe II and other singly-ionized iron-group elements are most important for the minimum of the modified Eddington luminosity, many other ions (including Fe III at higher temperatures) also contribute.

Davidson: A pair of mildly naïve heuristickal remarks --

(1) The basic idea of a temperature-dependent modified Eddington limit, with an opacity crisis (= recombination crisis) as a possible cause for LBV eruptions, is one of the oldest items in our modern LBV lore, antedating the term LBV. In a semipopular-level review article written in 1983 (Science 223, 243), Roberta and I were careful to include a qualitative description of this intuitive idea, which was then a dozen years old for one LBV, at least -- need I say which?! So, while it has been disappointing that sophisticated calculations have taken such a long time to follow naive intuition, it is also gratifying that analyses like those by Kudritzki's group seem 
to be vindicating the basic idea so far. Their two-mode results so closely resemble what some of us have been expecting/hoping-for, that it seems to me that nature is playing a joke on us if they are not on the right track. (In saying this, of course I am being sloppy about which source of opacity is the main culprit.)

(2) Here's a related empirical remark. Recently I calculated a few old-fashioned, simplified static atmospheres with plausible grey-opacity dependences and with a mass-luminosity relation appropriate for LBV's: pocket-calculator work. Suppose we characterize each of these idealized models by its maximum value of $\Gamma=$ $\left|g_{\mathrm{rad}} / g_{\mathrm{grav}}\right|$, which in the parameter range of interest occurs at some optical depth of order unity-- let's ignore the normal winds for my purposes! Then it turns out that the observed Upper Limit of stars in the $H-R$ diagram nicely follows the locus of $\Gamma_{\max } \approx 0.8$ (or maybe 0.7 or 0.9 , but not 1.0 , whose locus is distinctly different). Two poster papers only a meter apart upstairs, one by the Munichers and one by Nieuwenhuijzen and de Jager, evoke this same conjectural point when viewed together, if one is in the right frame of mind. Is there a critical value of the idealized $\Gamma_{\max }$ defined this way, marking the onset of instability?

In any case, we need some calculations aimed specifically at isolating the hypothetical instability -- analyses that include only those processes that are necessary for the instability (if it exists). Sophisticated NLTE atmosphere-and-wind models like those described by the Munich and JILA groups are valuable in demonstrating that interesting effects do indeed occur, but their industrial-strength levels of detail cause some distraction from the immediate problem. It seems unlikely that details of line formation, departures from LTE, etc., are crucial to the LBV instability. I don't know whether a largely analytic stability analysis or hydro runs on a big computer would be more appropriate, but in either case I suspect that simplified opacity dependences and simplified radiative transfer would suffice, and that the hydrodynamics are more important. Some work along these lines should have been inspired by Appenzeller's excellent discussions at two meetings in 1985--1986.

Immo Appenzel1er

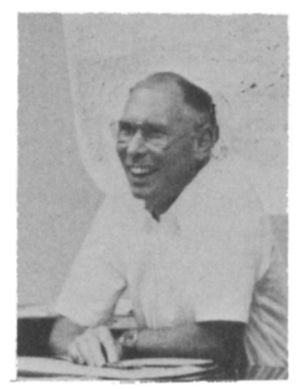

